\title{
The COMPARES Project: COnnectionist Methods for Preprocessing and Analysis of REmote Sensing data
}

\author{
J. Austin ${ }^{1}$, G. Giacinto ${ }^{2}$, I. Kanellopoulos ${ }^{3}$, K. Lees $^{1}$, F. Roli ${ }^{2}$, G. Vernazza ${ }^{2}$ \\ and G. Wilkinson $\left.{ }^{4}{ }^{*}\right)$ \\ 1 University of York, UK \\ 2 University of Cagliari, Sardinia, Italy \\ 3 EC Joint Research Centre, Ispra, Italy \\ 4 Kingston University, UK \\ $\left({ }^{*}\right)$ Formerly at EC Joint Research Centre, Ispra, Italy
}

\begin{abstract}
The European Concerted Action "COMPARES" (Concerted Action on COnnectionist Methods for Preprocessing and Analysis of REmote Sensing Data) was funded within the Environment and Climate Programme of the European Commission. The "COMPARES" project was aimed at focussing and coordinating future research at European level on the general theme of connectionist computation in the field of Earth Observation (EO). Connectionist computation can be interpreted as the use of artificial neural networks and parallel distributed processing systems.
\end{abstract}

\section{Introduction}

The overall objectives of the "COMPARES" Concerted Action were to:

1. assess what has been done so far throughout the European Union primarily (and to a lesser extent elsewhere) on the application of neural or connectionist computing in remote sensing;

2. evaluate the potential benefits to be derived from pursuing further research and development on this theme in a coordinated manner;

3 . draw up an appropriate research agenda for the future taking into account the results and conclusions of 1 . and 2 .

The Concerted Action was proposed to the European Commission in 1995 by a consortium of three partner institutions: the University of York, UK, (Department of Computer Science); the University of Cagliari, Sardinia, Italy (Department of Electrical and Electronic Engineering); and the EC Joint Research Centre, Ispra, Italy (Space Applications Institute). The Action was accepted for funding in early 1996 and work officially began on 1st March 1996 with an overall duration of 12 months. The Action was proposed as a contribution to Methodological Research related to space techniques within the Environment and Climate Programme (specifically section 3.1 .1 of the programme which includes 
the development of generic tools and techniques for automated preprocessing, interpretation and integration of remotely sensed and ground data).

In order to achieve the objectives as stated above, two main events were planned within the scope of COMPARES. The first event of this Concerted Action was an open workshop on neural network computing in remote sensing which was held at the University of York, UK, in July 1996. The aim of this workshop was primarily to obtain a current "snapshot" of the state of connectionist computing research in remote sensing. The second event of the Concerted Action was the Research Strategy Meeting held in Cagliari, Sardinia, in October 1996. In contrast to the York workshop, the Cagliari meeting was smaller and consisted only of a restricted group of invited experts who were primarily charged with the duty of identifying priorities for future research as input to deliberations on future funding within European programmes. In order to ensure Europe-wide participation in the events, individual "National Points of Contact" (NPOCs) were established in each European Union member state with a brief to identify and invite national experts to the two events.

In the framework of the project, five key research themes have been identified, which are believed to represent the overall findings and which were perceived by the partners as addressing particular technical problems in Earth observation besides being commercially significant. Detailed expositions of the five key research themes (including important sub-topics) are given in section 3 inclusive in this paper. Before beginning this exposition, however, it is useful to set the scene by examining the general background of connectionist computing in Earth observation to date, as revealed by the open remote sensing literature in general, and by the presentations given at the York workshop in particular.

\section{Connectionist Techniques in Remote Sensing Data Analysis}

\subsection{History of Connectionism}

The connectionist paradigm is a relatively recent development in computer science which owes much to the pioneering work of a small number of researchers[13]. The application of neural network systems to real world problems rose steadily during the 1980 s as progressively more people working on pattern recognition became aware of their existence and potential as an alternative to the more traditional approaches. There are now a large number of neural network models in use, differing in structure and training mechanisms. Some are more suited to unsupervised pattern recognition, others to supervised pattern recognition. Descriptions of the different systems can now be obtained from numerous texts, e.g. [4,5]. However, it is also well recognized that, the behaviour of a particular trained network, which is dictated primarily by the large set of numerical "weights" attached to the links between its individual internal processing units, is far from transparent. This aspect has certainly retarded the widespread acceptance of neural networks in many applications, remote sensing being no exception to this as identified in the course of this Concerted Action (see section 3.3 below). 


\subsection{The Rise of Connectionism in Earth Observation}

In EO, the earliest work on the use of neural networks took place around 1988 with the first papers appearing in peer-reviewed journals in 1989 and 1990 (e.g. work by Key et al. [6], Benediktsson et al. [7]). Once the idea had spread, the number of papers grew rapidly with several dozen publications per year by the mid 1990s. This trend appears to be continuing with an apparent year-on-year exponential rise in the number of researchers applying neural network algorithms to problems in EO. One of the more worrying aspects of this growth, however, is the seemingly incessant duplication of effort in testing approaches which have already been proven by others. The need to focus future European research on the most critical, and as yet unexplored, issues has been one of the reasons for the "COMPARES" Concerted Action.

\section{Research Themes Addressed in the COMPARES Project}

\subsection{Classification of Earth Observation Data}

General Problems The classification of multispectral image data to produce "thematic maps" has been one of the main uses of neural networks in Earth observation to date. Whilst neural network approaches have often been shown to yield "good" results in classification, overall a review of the literature [8] shows that there has been only a marginal improvement, if any, compared to the use of other more conventional approaches. Typical overall classification accuracies obtained from neural networks rarely exceed $80-85 \%$ under optimal conditions.

Potential Solutions and Research Priorities Although there is little evidence for widespread improvement in classification accuracy by use of connectionist approaches, it is difficult to make any reliable or conclusive statement on this because most reported experiments use completely different data sets. Conclusive evidence about the comparative value of connectionist approaches versus any other approach requires the production of public domain standard benchmark data sets. The assessment of classification accuracy is also an area for further development. A wider range of quality metrics and objective criteria need to be developed especially for comparing classifications made with significantly different numbers of classes or when sub-pixel or mixed class information is extracted. There should also be more research on the user's concept of what constitutes a good enough classification accuracy. Some recent research has also demonstrated that performance gains can be made by integrating connectionist and non-connectionist paradigms in classification [9]. In addition, it is now generally accepted that for many EO applications, it is essential to augment the remotely sensed data with ancillary geographical or contextual information and also with background human knowledge. 


\subsection{Signal Inversion in Earth Observation}

General Problems The retrieval of physical parameters describing land / ocean surfaces and the atmosphere from satellite observations is often non-trivial. The quality of existing satellite transfer functions or inverse models is often inadequate for retrieving parameters at a high enough precision to be used in environmental models. This problem is especially critical for global scale atmospheric and oceanic circulation models, numerical weather prediction models and models of land surface processes and their long term changes.

Potential Solutions and Research Priorities Signal inversion can be viewed as a mathematical transformation from one representation space to another. In this sense it is directly analogous to classification, the main difference being that classification is aimed at the retrieval of fixed class labels rather than continuous parameters. So far the uses of neural networks for inversion in Earth observation have been few, though some experiments have been reported [10,11]. Connectionist approaches could be used to create non-linear numerical models of environmental phenomena. [12] and also to model sensor characteristics as part of the overall signal inversion process.

\subsection{Usability of Advanced Software Systems in Earth Observation Data Analysis}

General Problems The use of many software systems designed for the analysis of Earth observation data continues to be difficult for many value added product suppliers and for the majority of potential end users. They generally require months of training and experience for effective use and rarely offer advanced algorithms such as neural networks or other innovative pattern recognition techniques. A further major problem in the uptake of connectionist methods by remote sensing data users is that neural networks are not easily used by nonexperts and their internal behaviour is not transparent $[13,14]$.

Potential Solutions and Research Priorities At a general level there needs to be more development of intelligent front ends (IFEs) for software tools used in EO. More advanced - yet easy to use - visualization tools are needed to help users understand patterns in data, particularly to include the time dimension so that long term trends in regional or global land cover can be easily understood and presented to decision makers and the general public. Ideally the next generation of software systems for EO should make informed decisions on behalf of the user. In the connectionist context this must include automated parameter setting and control of training as minimum requirements. Moreover online assistance to "engineer" a neural network architecture to the desired application need would be highly valuable. 


\subsection{Scalability and "Large" Problem Solving in Remote Sensing}

General Problems Observations at regional and global scales are more cost effective than ground based gathering of data and can reveal patterns of environmental change of major significance for mankind. However, the use of remote sensing for regional to global scale mapping or monitoring is actually relatively unreliable and error prone. A second scale-related problem is the need in many current EO applications to analyse very large data volumes, The current trends towards significantly increased numbers of spectral channels and very high spatial resolution multispectral imagery points to a need for very high speed processing e.g. via special purpose computer architectures.

Potential Solutions and Research Priorities In order to map or monitor "large" areas by EO, it is necessary to develop classification techniques which are "portable", i.e. they are adaptable and perform well in different geographical contexts. The potential of connectionist classifiers to provide a solution to this problem needs to be investigated. An advantage of connectionist systems is that they can be incrementally expanded to solve increasingly complex problems by adding additional processing units or layers. One of the most difficult problems will be the training of very large networks or ensembles of sub-nets. Recent work on minimizing training sets (e.g. $[15,16])$ needs to be continued and expanded with a new emphasis on training very large neural systems. Efforts to improve training or learning for large or complex connectionist systems will also be of benefit in the analysis of very high resolution imagery, where there is potential to develop complex connectionist systems to detect structural features as part of "image understanding" for topographic mapping applications.

\subsection{The Impact of Emerging Systems Technologies: Telematics, the Internet, and Distributed Processing}

General Problems There is a general trend in present day information systems towards distributed environments and remote access to data and processes via "telematics" technologies. Although many EO data archives are distributed between different data providers, most processing of EO data is carried out with large locally resident software packages. This is relatively inefficient as users may require special processing modules on very rare occasions. Improvements are needed in EO to implement fully distributed processing following the clientserver paradigm with the possibility to down-load both data and processing modules from globally distributed providers e.g. via the Internet.

Potential Solutions and Research Priorities Since connectionist algorithms can be adapted to many different problems in remote sensing, a few basic software modules could be used for multiple purposes, providing economies in code production. Blocks of generic neural network code may be downloadable over 
the Internet in the same way as data sets. Moreover, weight sets for neural algorithms may be attached as metadata to EO data within an object-oriented system. The recent trend towards the use of the JAVA language to encode "applets" embedded within information provided on the World Wide Web needs exploring as a model for the distribution and analysis of EO data. Developments are still needed on security and charging mechanisms for data and procedure brokering over the Internet.

\section{Main Achievements of the COMPARES Project}

The previous section has identified a number of potential avenues for future research related to the use of neural networks in Earth observation. Some of these involve fundamental issues related to neural networks which are not specific to remote sensing. Others are very specific to remote sensing or to the way in which remote sensing data is exploited or integrated into broader environmental information systems.

In order to sum up the findings of this Concerted Action, the main topics for future research are highlighted in the list below. Although all topics identified in subsections 3.1-3.5 are considered important and meriting further study, priorities for future action have been allocated to these topics on a scale of 1-4 as follows:

Priority 1: Essential research topics; mostly on innovative uses of connectionist methods in $\mathrm{EO}$; probably vital to many $\mathrm{EO}$ applications besides having scope to open up significant new markets for EO; should be undertaken with urgency:

1. use of connectionist systems to model environmental processes

2. use of connectionist systems for complex / non-linear signal inversion problems in $\mathrm{EO}$

3. use of connectionist methods for geometrical operations on EO data (especially very high resolution data)

4. use of connectionist systems for structural feature detection

5. integration of human contextual knowledge in EO data analysis by connectionist methods

6. integration of ancillary geographical information in EO data analysis by connectionist methods

7. role of connectionist processing in distributed / telematic EO frameworks (code re-use, "neural applets" etc.)

8. assessment of the requirements for high-performance hardware support in the use of connectionist methods for $\mathrm{EO}$

9. soft classification approaches e.g. neurofuzzy systems

10. establishment of easy-access benchmark data sets (with ground truth) for objective comparisons of classified performance

Priority 2: Important research topics; will help to consolidate use of connectionist methods in EO; could probably enhance the value of $\mathrm{EO}$ data and the market for $\mathrm{EO}$ related services in a number of sectors: 
1. improved quality metrics for EO products especially from connectionist systems and "good enough" product definition

2. hybrid connectionist / non-connectionist classifiers

3. development of "best practice" for connectionist systems in EO

4. studies of the feasibility of modular very large neural systems for large scale problems, to include addressing the issue of scalability of various network architectures (hyperspectral data analysis, pan-continental mapping etc.)

5. training set minimization for large connectionist systems

6 . investigation of alternative charging systems for network distribution of connectionist and non-connectionist applications resources (e.g. "applets" via the Internet)

Priority 3: Potentially valuable research topics which could assist in the development and use of advanced algorithms in EO; may help to extend the EO market to a moderate degree:

1. visualization tools to aid understanding of data and behaviour or connectionist systems

2. visual programming interfaces for constructing connectionist systems

3. automated neural network system prototyping tools

4. studies of the relationship between connectionist classification and spectral mixture analysis

5. use of consensus theory to integrate parallel connectionist systems

Priority 4: Research is needed as there is potential to improve EO products and EO uptake, though these topics are not critical in the majority of EO applications:

1. transparent mapping of applications onto parallel neural hardware systems

2. investigate methods for improved training of connectionist systems

3. exploration of novel / unconventional neural network models for EO data analysis

More details about the COMPARES Project can be found at the WWW address: http://thalassa.jrc.it/compares/comp-home.html

\section{Acknowledgements}

The authors wish to acknowledge the support of the European Commission's Environment and Climate Programme for the COMPARES Concerted Action (contract number ERB ENV4-CT95-0151). The authors are also particularly grateful to Dr. A. Cross (EC, DGXII-D-4, Brussels), monitoring officer for COMPARES, for his constructive advice on the formulation and execution of this Concerted Action. Thanks are also due to the many staff and colleagues at the University of Cagliari, the University of York, and the EC Joint Research Centre, and also to the National Points of Contact from each EU Member State plus the participants, who directly or indirectly contributed to the success of the COMPARES activities. 


\section{References}

1. Kohonen, T. 1984. Self Organization and Associative Memory. Springer Series in Information Sciences, Vol. 8. Springer-Verlag. (Also 2nd. edition, 1988).

2. Grossberg, S. 1976. Adaptive pattern recognition and universal recoding: Part 1. Parallel development and coding of neural feature detectors, Biological Cybernetics, 23, 121-134.

3. Rumelhart, D. E., McClelland, J. L. and the PDP Research Group. 1986.Parallel Distributed Processing, Explorations in the Microstructure of Cognition, Vol. 1: Foundations, MIT Press, Cambridge, Massachusetts.

4. Pao, Y. -H. 1989. Adaptive Pattern Recognition and Neural Networks, AddisonWesley, Reading, Massachusetts.

5. Dayhoff, J. 1990. Neural Network Architectures. (New York: Van Nostrand Reinhold), 259pp.

6. Key, J., Maslanic, A. and Schweiger, A. J. 1989. Classification of merged AVHRR and SMMR Arctic data with neural networks, Photogrammetric Engineering and Remote Sensing, 55, 9, 1331-1338.

7. Benediktsson, J. A., Swain, P. H., and Ersoy, O. K. 1990 . Neural network approaches versus statistical methods in classification of multisource remote sensing data, IEEE Transactions on Geoscience and Remote Sensing, 28, 4, 540-552.

8. Wilkinson, G. G. 1997. Neurocomputing for Earth observation - recent developments and future challenges, chapter 16 in 'Recent Developments in Spatial Analysis -Spatial Statistics, Behavioural Modelling and Neurocomputing', M. M. Fischer and A. Getis (editors), Berlin, Springer-Verlag.

9. Wilkinson, G. G., Fierens, F. and Kanellopoulos, I. 1995. Integration of neural and statistical approaches in spatial data classification, Geographical Systems, 2, 1-20.

10. Pierce, L.E., Sarabandi, K., and Ulaby, F.T. 1994. Application of an artificial neural network in canopy scattering inversion, International Journal of Remote Sensing, $15,16,3263-3270$.

11. Chen, K.S., Kao, W.L., and Tzeng, Y.C. 1995. Retrieval of surface parameters using dynamic learning neural network, International Journal of Remote Sensing, $16,5,801-809$.

12. Seginer, I., Boulard, T., and Bailey, B.J. 1994. Neural network models of the greenhouse climate, Journal of Agricultural Engineering Research, 59, 203-216.

13. Serpico, S.B. and Roli, F. 1995. Classification of multisensor remote-sensing images by structured neural networks, IEEE Trans. Geoscience and Remote Sensing, 33, $3,562-578$.

14. F.Roli, S.B.Serpico, and G.Vernazza, Neural Networks for classification of remotely sensed images, Part 2, Chapter 15, Fuzzy Logic and Neural Network Handbook, McGraw-Hill Pub., C.H.Chen Editor, 1996

15. Foody, G. M., McCulloch, M. B., and Yates, W. B. 1995. The effect of training set size and composition on artificial neural network classification, International Journal of Remote Sensing, 16, 9, 1707-1723.

16. Hepner, G. F., Logan, T., Ritter, N., and Bryant, N. 1990. Artificial neural network classification using a minimal training set: comparison to conventional supervised classification, Photogrammetric Engineering and Remote Sensing, 56, 4, 469-473. 